\title{
Characteristics of Electroless Sn Plating Electrolyte using a Choline Chloride-Based Ionic Liquid
}

\author{
Chieon Park ${ }^{1,2}$, Bongyoung Yoo $^{2}$, and Joonkyun Lee ${ }^{1, *}$ \\ ${ }^{I}$ Smart Textiles R\&D Group, Research Institute of Industrial Technology Convergence, Korea Institute of Industrial Technology, \\ Ansan 15588, Republic of Korea \\ ${ }^{2}$ Department of Materials Science and Chemical Engineering, Hanyang University, Ansan 15588, Republic of Korea
}

\begin{abstract}
In the present study, a choline chloride(ChCl)-based ionic liquid(IL) was used as an electrolyte to confirm the characteristics of electroless tin plating. The effect of the type of additive and reducing agent, as well as the addition of complexing agent and water, on the properties of the ChCl-based IL, including the freezing point, viscosity, conductivity, open circuit potential, and plating thickness, was investigated. The results showed that the crystallization temperature was $25{ }^{\circ} \mathrm{C}$ when the ChCl-thiourea molar ratio was $1: 1$, and it decreased to $10^{\circ} \mathrm{C}$ upon adding the additive, while the conductivity increased and viscosity decreased. Dimethylthiourea was added to promote the electroless plating reaction. Electrolytic tin plating was performed at $70{ }^{\circ} \mathrm{C}$ using $\mathrm{ChCl}$-thiourea-based IL as the electrolyte. The thickness of the tin-plated layer was $1.5 \mathrm{\mu m}$ when malonic acid and dimethylthiourea were used. The addition of a small amount of water increased the plating speed and thickness, and the maximum thickness of $2 \mu \mathrm{m}$ for the tin-plated layer was obtained after $30 \mathrm{~min}$. These characteristics demonstrate the potential applications of these environmentally friendly electrolytes for fast, thick, and dense electroless tin plating using ILs at low temperatures.
\end{abstract}

(Received May 4, 2018; Accepted July 2, 2018)

Keywords: ionic liquid, Sn plating, electroless plating, choline chloride, deep eutectic

\section{INTRODUCTION}

The presence of lead both in electronic components and in automotive electronics not only induces skin disease and various types of cancer, but can also cause serious global environmental pollution. Indeed, the Restriction of Hazardous Substances Directive RoHS(Restriction of Hazardous Substances) originating from the European Union (EU) prohibits the use of six harmful substances in electronic components, of which lead is given as one example. As such, to replace lead in such components, the development of environmentally friendly tin plating techniques is required. To date, electroless tin plating technology has been applied to many industrial fields, including in the production of semiconductor packaging, printed circuit boards, and other electrical components. Thus, the development of this technique has received growing interest in recent years.

*Corresponding Author: Joonkyun Lee [Tel: +82-31-8040-6838, E-mail: leejk@kitech.re.kr] Copyright (c) The Korean Institute of Metals and Materials
Printed circuit boards (PCBs) and package substrates have become increasingly smaller and denser. Accordingly, substitution plating such as electroless tin which can be finely treated is widely used in the precision industry. Despite the many advantages of displacement plating, low plating thicknesses (0.7-1.0 microns) were a problem. Therefore, this study was studied to solve this problem.

Electroless plating refers to a reaction in which the oxidation of a reducing agent and the reduction of metal ions occur simultaneously on the surface. In this process, the electrolyte tends to contain a reducing agent, a complexing agent, a surfactant, a $\mathrm{pH}$ adjuster, and a stabilizer [1,2]. Electroless tin plating can be carried out under either or acidic or alkaline conditions. The use of alkaline conditions results in the formation of a $\mathrm{Sn}$ layer by disproportionation, while under acidic conditions, the $\mathrm{Sn}$ is deposited by a displacement reaction that forms a $\mathrm{Cu}$-thiourea complex. Currently, the most well-known electroless tin plating technique involves the displacement of $\mathrm{Cu}$ by $\mathrm{Sn}$ in a $\mathrm{Sn}-$ thiourea acidic bath. Indeed, this technique has received 
significant attention due to its ability to form uniform surfaces, and to achieve successful plating even at low temperatures $[3,4]$.

Furthermore, thickening of the $\mathrm{Sn}$ plating has been proposed to improve solderability, surface flatness, and pitch compatibility, while suppressing the growth of Sn whiskers. In this context, the reliability of the whole product, uniform, dense, thick plating is required, and the plating rate is increased, studies based on increasing the thickness of the Sn plate during the immersion plating process are of particular importance [6].

Indeed, to overcome the issues outlined above, particular attention has also been directed to the use of ionic liquids, which are considered environmentally friendly materials for next-generation electrolytes [7]. When compared with other organic solutions, ILs exhibit low toxicity and high dissolvability for both organic and inorganic substances due to their high polarity. In addition, ILs are non-volatile due to their essential lack of steam pressure. Furthermore, ILs demonstrate high ion conductivities, broad electrochemical ranges, and high thermal and chemical stabilities at high temperatures, thereby resulting in increased attention for their application as green solvents [8,9].

The properties of ILs depend mainly on the size and structure of the anions and cations composing the IL. Indeed, ILs are known as "Designer Solvents," as their design can be altered depending on the intended purpose, and so significant research into ILs as replacement solvents and electrolytes for electrochemical processes is being carried out actively in both research laboratories and in industry $[10,11]$. Due to their highly hydrophilic nature, ILs tend to absorb moisture readily from their surroundings. This absorption of water thereby leads of a decrease in viscosity and an increase in electrical conductivity, although the absorption of excess water considerably reduces the electrochemical window [12]. In addition, the absorbed water molecules also induce the decomposition of ILs, thereby leading to unwanted side reactions and corrosion of the connecting metal [13]. However, recent reports have suggested that the use of an electrolyte prepared with the addition of a small amount of water to the IL has a beneficial effect on the morphology of the plate [14]. Indeed, the corresponding reduction in viscosity of the IL and increase in both electrical conductivity and current efficiency at relatively low temperatures through the promotion of ionic dissociation are beneficial in the context of IL applicability [15].

According to a recent review, when water was added to an IL prepared using ChCl-2Urea, water bonded with the urea molecules rather than with $\mathrm{ChCl}$. In addition, the dissociation of water was suppressed by the strong hydrogen bonds present in this system, thereby maintaining a wide electrochemical window. As reported, upon the addition of water molecules to the ChCl-2Urea-based IL, urea is replaced with water molecules, which in turn produces additional free $\mathrm{Ch}^{+}$ions, thereby increasing the extent of ionic dissociation [15].

Thus, we herein investigated the applicability of an IL, synthesized using $\mathrm{ChCl}$, urea, and thiourea, as an environmentally friendly electrolyte for use in a tin plating technique, as this IL is expected to exhibit a high ion conductivity and stable electrochemical properties.

\section{EXPEIMENTIAL}

\subsection{Materials}

To suppress the hygroscopic properties of Choline chloride(Sigma-Aldrich, 98\% pure), a desiccator was employed for storage. Urea(Daejung, 98\% pure), thiourea (Daejung, 96\% pure), and dimethylthiourea (Sigma-Aldrich, 99\% pure) were used without additional pretreatment, where thiourea and dimethylthiourea were employed as a complexing agent and a complexing agent derivative, respectively. In addition, malonic acid(Acros, 99\% pure), oxalic acid(Acros, 99\% pure), succinic acid(Acros, 99\% pure), ethylene glycol(Shinyo, 99\% pure), ethylene alcohol(Duksan, 99.9\% pure), propylene glycol (SigmaAldrich, 99.5\% pure) and propyl alcohol (Sigma-Aldrich, $99.5 \%$ pure) were employed as additives. Furthermore, tin chloride(Sigma-Aldrich, 96\% pure), sodium phosphate (Sigma-Aldrich, 96\% pure), and titanium chloride(SigmaAldrich, $12 \%$ pure $\mathrm{TiCl}_{3}$ basis) were also used as obtained, with the latter two reagents being employed as reducing agents.

\subsection{Preparation of deep eutectic-based IL}

$\mathrm{ChCl}$ and urea $(\mathrm{U})$ were mixed in a 1:2 molar ratio, then 
stirred at $200 \mathrm{rpm}$ at $80{ }^{\circ} \mathrm{C}$ until the formation of the deep eutectic IL ChCl-U was complete. Using the same method, $\mathrm{ChCl}$ and thiourea (TU) were employed in a 1:1 molar ratio to produce an additional deep eutectic IL, i.e., $\mathrm{ChCl}$ TU.

\subsection{Characterization of the electrolyte}

To measure the crystallization temperatures of these prepared ILs, they were each placed in a beaker, heated to $80{ }^{\circ} \mathrm{C}$, then mixed by stirring at $200 \mathrm{rpm}$. Following the complete dissolution of each powder, a chiller was used to gradually lower the temperature, and the temperature at which crystals began to form was recorded. The Freezing point is the average results of 5 points.

Viscosity was measured using a digital viscometer (Brookfield DV2T, U.S.A) equipped with RV spindle no. 2 , and the speed was regulated to ensure that the torque was maintained between 90 and $100 \%$. For ionic conductivity measurements, a Multi-Range Conductivity Meter (Hanna HI8633, U.S.A) was employed. The open circuit potential (OCP) was measured using an electrochemical impedance spectroscopy (EIS) Potentiostat (EG CS310, S. Korea), with copper and platinum working electrodes, a $\mathrm{Ag} / \mathrm{AgCl}$ reference electrode, and a platinum wire counter electrode.

\subsection{Characterization of the immersion $\mathrm{Sn}$ coatings}

The electrolyte was prepared using the 1:1 ChCl-TU solution, tin chloride $(12 \mathrm{~g} / \mathrm{L}$, metal salt), thiourea $(40 \mathrm{~g} / \mathrm{L}$, complexing agent), dimethylthiourea $(40 \mathrm{~g} / \mathrm{L}$, complexing agent derivative), sodium phosphate, and titanium chloride (reducing agents). Electroless tin plating was then conducted at $70{ }^{\circ} \mathrm{C}$ over 30 mins using a Hull Cell and a $\mathrm{Cu}$ cathode was used pretreatment through degreasing, etching, and washing, and the effects of plating time and the contents of water and the reducing agent on the final plating thickness were examined.

The thickness of Sn coatings was measured by Energy dispersive X-ray spectroscopy (XRF) (ISP 150WT, S. Korea)

Structure and morphology of Sn coatings were investigated by scanning electron microscope (SEM) (SEC SNE4500M, S. Korea) respectively.

\section{RESULT AND DISCUSSION}

\subsection{Freezing point of the ILs}

The freezing points of the ILs prepared by mixing $\mathrm{ChCl}$, urea, and thiourea at two different molar ratios (i.e., 1:1 and 1:2) are outlined in Fig. 1. As indicated, the lowest freezing point of $\mathrm{ChCl}-\mathrm{U}$ was recorded at $12{ }^{\circ} \mathrm{C}$ for a $1: 2$ molar ratio, while that of $\mathrm{ChCl}-\mathrm{TU}$ was $25^{\circ} \mathrm{C}$ for a $1: 1$ molar ratio, thereby suggesting that these ILs are suitable for use as ambient temperature solvents. When considering lowering the freezing point of these ILs, interactions between the urea molecules and the $\mathrm{Cl}$ ions should be considered. Indeed, this coincides with the crystallographic data obtained for the solid adduct $\left[\left(\mathrm{CH}_{3}\right)_{3} \mathrm{~N}^{+} \mathrm{CH}_{2} \mathrm{CH}_{2} \mathrm{OH}^{-}\right]{ }_{2} \mathrm{C}_{2} \mathrm{O}_{4} 2^{-} \cdot 2\left(\mathrm{NH}_{2}\right)_{2} \mathrm{CS}$, which indicates the presence of an extensive hydrogen bonding network between the thiourea molecules and the oxalate anions [16].

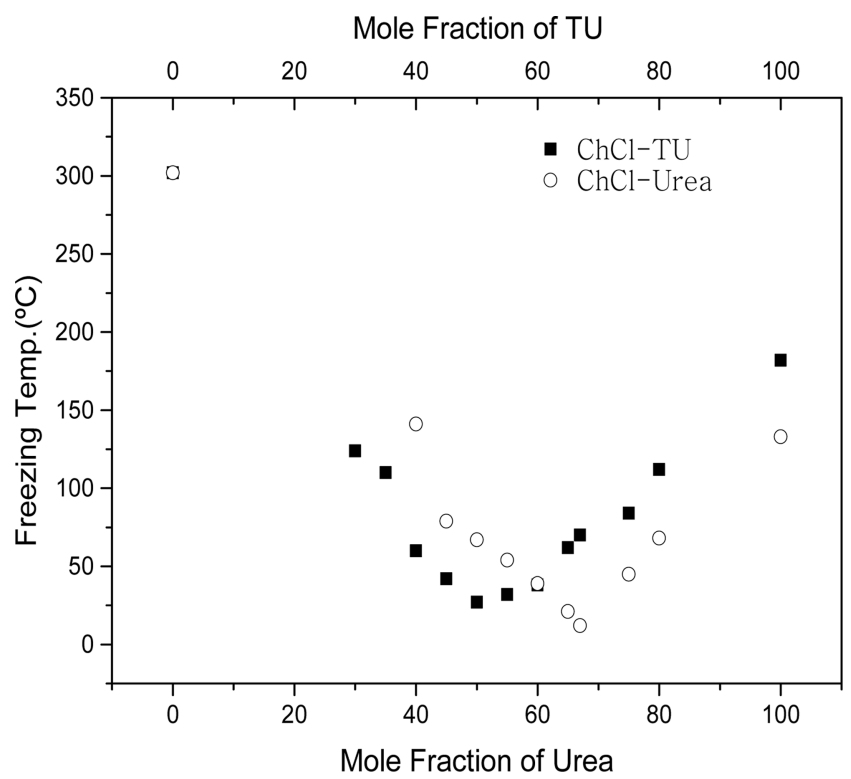

Fig. 1. Freezing point of $\mathrm{IL}(\mathrm{ChCl}-\mathrm{U})$ or $\mathrm{IL}(\mathrm{ChCl}-\mathrm{TU})$ as a function of composition.

To determine the effect of additives on the freezing points of these ILs, ethylene glycol, ethylene alcohol, propylene glycol, propyl alcohol, malonic acid, succinic acid, or oxalic acid $(0.5 \mathrm{~mol}$ in each case) was added to the IL, and the resulting freezing points were measured and the results listed in Table 1. 
Table 1. Determination of the freezing point with 1: 2 ratio of choline chloride / urea and 1: 1 choline chloride / thiourea according to additives.

\begin{tabular}{|c|c|c|}
\hline \multirow{2}{*}{$\begin{array}{c}\text { Additives } \\
\text { None additives }\end{array}$} & \multicolumn{2}{|c|}{$\begin{array}{l}\text { Freezing point } \\
\quad \mathrm{T}_{\mathrm{f}} /{ }^{\circ} \mathrm{C}\end{array}$} \\
\hline & $\begin{array}{c}\text { ChCl-U } \\
12\end{array}$ & $\begin{array}{c}\text { ChCl-TU } \\
25\end{array}$ \\
\hline Ethylene glycol, $\mathrm{C}_{2} \mathrm{H}_{6} \mathrm{O}_{2}$ & 5 & 10 \\
\hline Ethylene alcohol, $\mathrm{C}_{2} \mathrm{H}_{6} \mathrm{O}$ & 10 & 22 \\
\hline Propylene glycol, $\mathrm{C}_{3} \mathrm{H}_{8} \mathrm{C}_{2}$ & 9 & 23 \\
\hline Propyl alcohol, $\mathrm{C}_{3} \mathrm{H}_{8} \mathrm{O}$ & 11 & 25 \\
\hline Malonic acid, $\mathrm{C}_{3} \mathrm{H}_{4} \mathrm{O}_{4}$ & 5 & 12 \\
\hline Succinic acid, $\mathrm{C}_{4} \mathrm{H}_{6} \mathrm{O}_{4}$ & 9 & 20 \\
\hline Oxalic acid, $\mathrm{C}_{2} \mathrm{H}_{2} \mathrm{O}_{4}$ & 8 & 13 \\
\hline
\end{tabular}

\subsection{Characterization of the electrolyte}

The conductivities and viscosities of the ILs containing 0.5 mol malonic acid, ethylene glycol, or oxalic acid were also measured, and the results are shown in Fig. 2. Interestingly, the lowest viscosity $(254 \mathrm{cP})$ and highest conductivity $(2080 \mathrm{mS} / \mathrm{cm})$ were achieved in the presence of malonic acid. In addition, as shown in Fig. 2, when ionic bonds are formed, the correlation between viscosity $(\eta)$ and conductivity $(\sigma)$ can be explained using the Stokes-Einstein equation and the Nernst-Einstein equation as follows:

$$
\begin{aligned}
& \mathrm{D}=\frac{k T}{c \pi \eta r} \\
& \sigma=\frac{N e^{2}}{k T}\left(D_{+}+D_{-}\right) \alpha
\end{aligned}
$$

where $\mathrm{D}$ is the self-diffusion coefficient of the ion, $\mathrm{k}$ is the

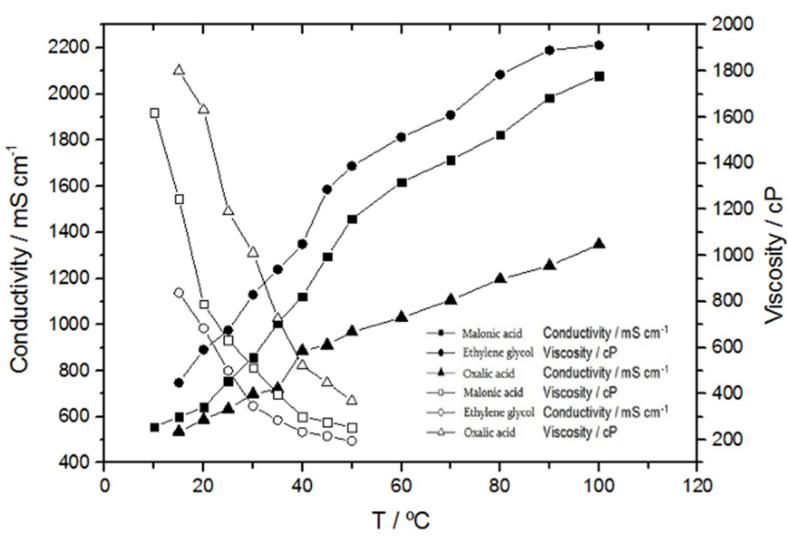

Fig. 2 Conductivity and viscosity of additives in a IL(ChCl-TU) as a function of temperature.
Boltzmann constant, $\mathrm{T}$ is the temperature, $\mathrm{c}$ is a coefficient determined by the boundary conditions, $r$ is the Stokes radius of the ion, $\mathrm{N}$ is the Avogadro constant, and e is the charge of an electron. In addition, $\alpha$ indicates the degree of dissociation, and the conductivity, $\sigma$, is determined from the degree of dissociation and viscosity.

Based on thermodynamic principles, no reaction occurs between $\mathrm{Cu}$ and $\mathrm{Sn}^{2+}$, and so the addition of complexing agents, such as thiourea, is necessary to decrease the equilibrium electrode potential of $\mathrm{Cu}$ to a level lower than that of Sn. More specifically, the presence of thiourea induces the formation of a $\mathrm{Cu}(\mathrm{Tu})_{4}{ }^{+}$complex that has a low electrode potential, thereby allowing for a displacement reaction to occur [6]. Following completion of the displacement reaction, Sn ions are reduced by reducing agents, thereby allowing the electroless tin plating process to take place.

To analyze the feasibility of this technique, the OCP was measured to determine changes in the electrode potential of $\mathrm{Cu}$ upon the addition of thiourea and $\mathrm{ChCl}$. As indicated in Fig. 3, an increase in the concentration of either thiourea or $\mathrm{ChCl}$ was effective in decreasing the OCP of $\mathrm{Cu}$. Similarly, the OCP changed upon the addition of dimethylthiourea as a complexing agent derivative. Furthermore, an increase in the concentrations of thiourea and dimethylthiourea resulted in decreased electrolyte OCP values. More specifically, as indicated in Fig. 4, upon the addition of $>40 \mathrm{~g} / \mathrm{L}$ thiourea and dimethylthiourea, the OCP decreased significantly after a period of relative stability.

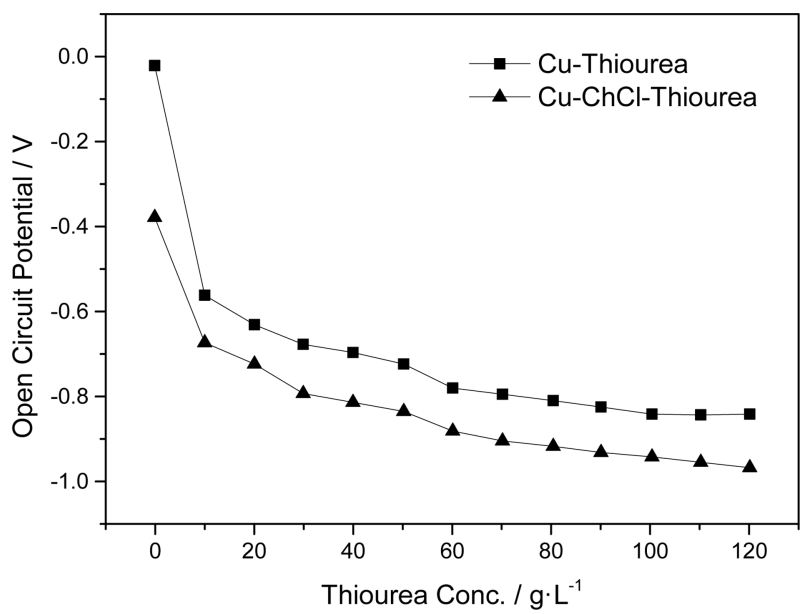

Fig. 3. Effect of Thiourea concentration on $\mathrm{OCP}$ of $\mathrm{Cu}$ working electrode (square) and Thiourea in $\mathrm{ChCl}$ solution (triangle), respectively, at room temperature. 


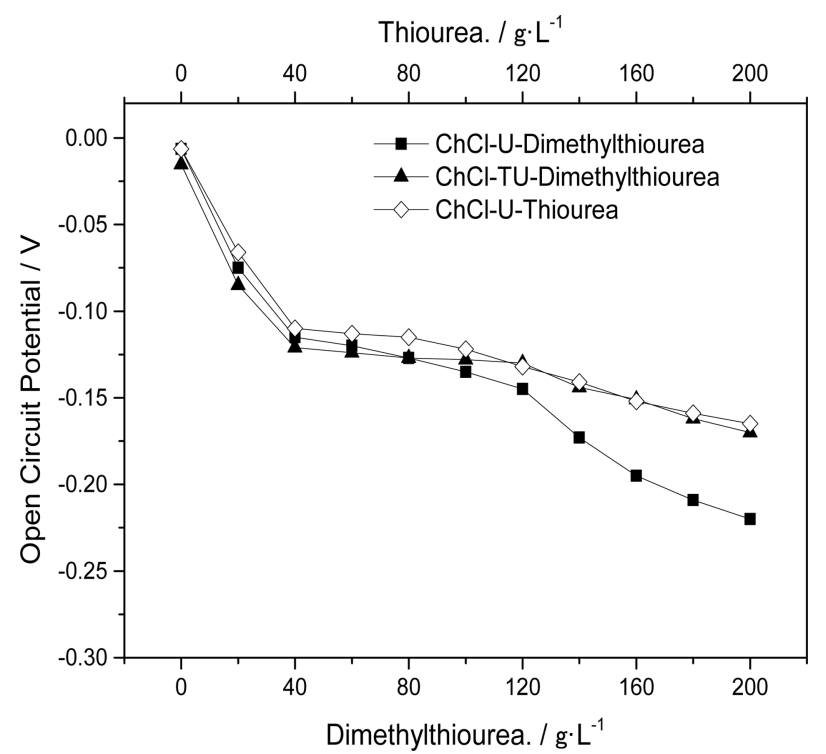

Fig. 4. Effect of Dimethylthiourea and thiourea concentration on OCP of Dimethylthiourea in $\mathrm{ChCl}-\mathrm{U}$ solution (solid), Dimethylthiourea in ChCl-TU solution (triangle), Thiourea in $\mathrm{ChCl}-\mathrm{U}$ solution (diamond) respectively, at room temperature.

\subsection{Characterization of the immersion $\mathrm{Sn}$ plating}

Electroless tin plating was then performed using an electrolyte prepared using the ChCl-TU IL, dimethylthiourea and thiourea as complexing agents, tin chloride as the metallic salt, the desired reducing agents, and water, and the variations in plating thickness were observed with respect to the plating time and the quantity of added reducing agents and water.

As shown in Fig. 5, the plating thickness increased when both sodium phosphate and titanium chloride were added, with the optimal thickness being observed with reducing agent concentrations of $0.2 \mathrm{~mol} / \mathrm{dm}^{3}$. However, at concentrations $>0.3 \mathrm{~mol} / \mathrm{dm}^{3}$, a significant reduction in the plating efficiency was observed. In addition, sodium phosphate was found to be more effective than titanium chloride in increasing the plating efficiency.

The addition of water was also found to have a positive influence on the plating efficiency, with the addition of $6 \mathrm{wt} \%$ water giving a maximum plating thickness of $2.03 \mu \mathrm{m}$. This may be explained by the decreased viscosity and increased conductivity resulting from the addition of water, which led to increased ionic dissociation [15]. In addition, as illustrated in Fig. 6, the thickness of the Sn

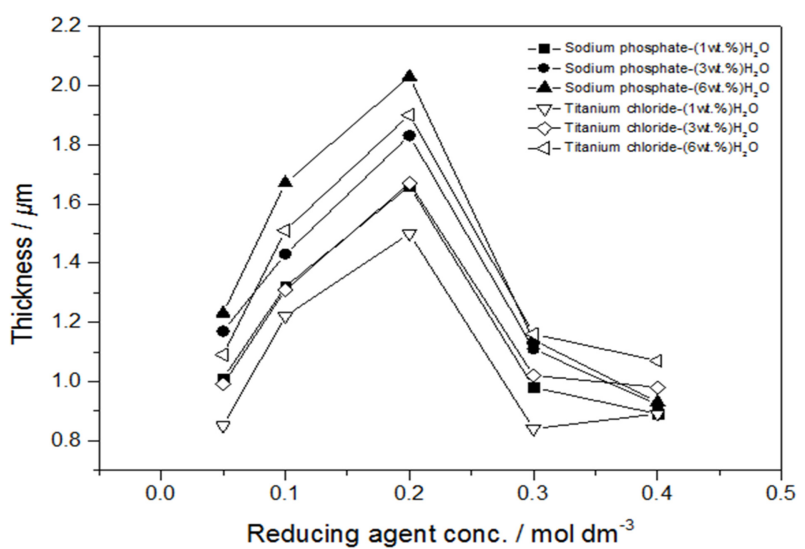

Fig. 5 Effect of reducing agent concentration on the thickness of immersion $\mathrm{Sn}$ coatings at water systems.

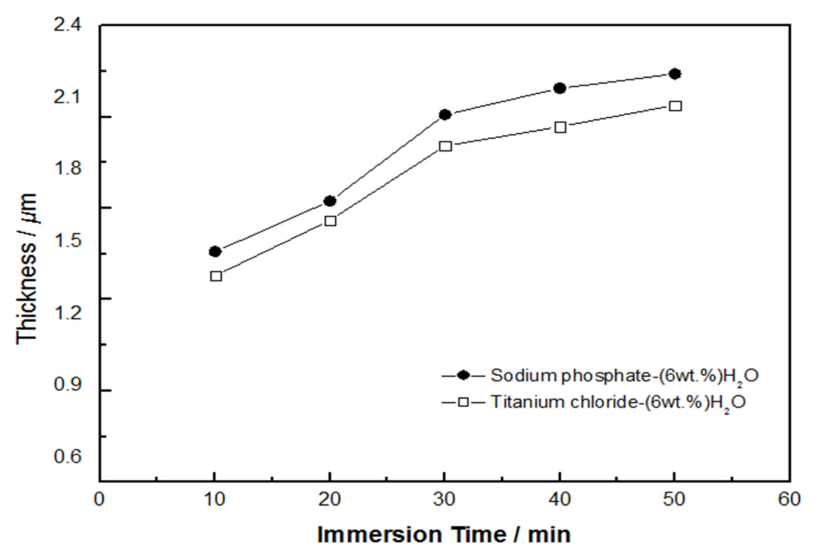

Fig. 6 Effect of dipping times on the thickness of immersion Sn coatings.

coating generally exhibited a positive correlation with increasing immersion time, with a gradual increase being observed after $30 \mathrm{~min}$. As such, the decrease in deposition rate after 30 min was attributed to reduced reactivity, as with longer immersion times, there is a higher possibility that $\mathrm{Sn}^{2+}$ comes into contact with the $\mathrm{Cu}$ surface [17].

The microscopic morphology of an Sn coating that had been plated using the IL electrolyte at $70{ }^{\circ} \mathrm{C}$ over $30 \mathrm{~min}$ is shown in Fig. 7. Interestingly, the surface morphology largely depended on the presence of both water and reducing agents during the plating process. In addition, as shown in Figs. 7a, $7 \mathrm{c}$, and $7 \mathrm{e}$, the surface of the Sn coating was relatively uniform and non-porous when reducing agents were employed.

Furthermore, the presence of water resulted in increased plating thicknesses and enhanced crystallinity in Sn, which 

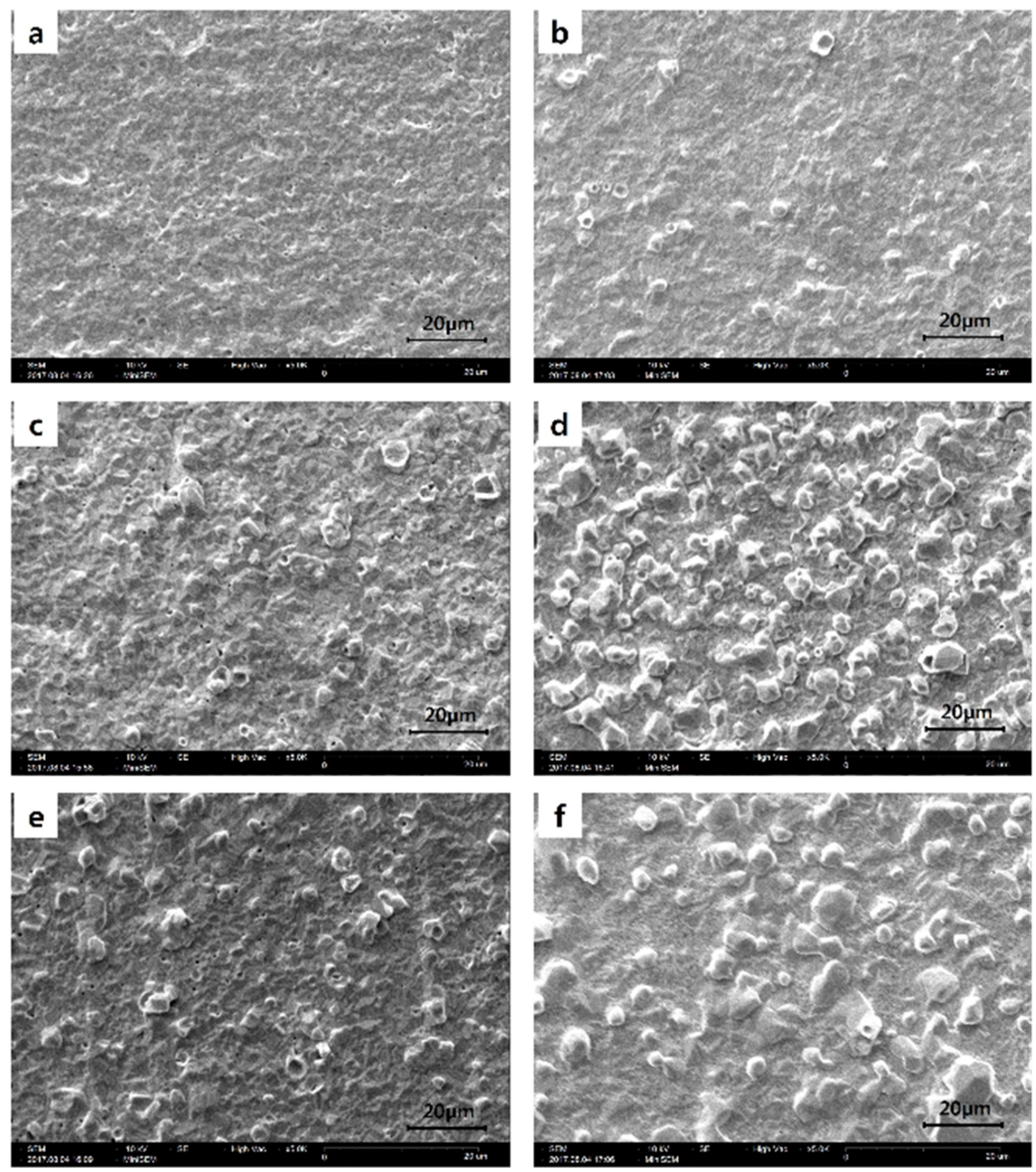

Fig. 7 Micrographs of the Sn coatings electroless plating from the ILs electrolytes containing (a) IL(ChCl-TU), (b) IL(ChCl-TU)-water $6 \mathrm{wt} \%$, (c) IL(ChCl-TU)-titanium chloride $0.2 \mathrm{~mol} / \mathrm{dm}^{3}$, (d) IL(ChCl-TU)-titanium chloride $0.2 \mathrm{~mol} / \mathrm{dm}^{3}$-water $6 \mathrm{wt} \%$, (e) IL(ChCl-TU)sodium phosphate $0.2 \mathrm{~mol} / \mathrm{dm}^{3}$, (f) IL(ChCl-TU)-sodium phosphate $0.2 \mathrm{~mol} / \mathrm{dm}^{3}$-water $6 \mathrm{wt} \%$ at $70{ }^{\circ} \mathrm{C}, 30 \mathrm{~min}$.

could be attributed to the reduced viscosity of the IL and the greater extent of ionic dissociation.

\section{CONCLUSIONS}

Following the formation of the deep eutectic IL using $\mathrm{ChCl}$ and thiourea, charge transport in the IL was investigated, and this was also found to be affected by both the ionic dissociation and viscosity. Finally, investigation of the freezing point of the malonic acid-containing IL confirmed that the IL could exist as liquid at room temperature due to hydrogen bonding between $\mathrm{ChCl}$ and thiourea.
It therefore appears that the presence of thiourea in the IL electrolyte results in the equilibrium electrode potential of $\mathrm{Cu}$ becoming lower than that of Sn, consequently enabling the desired electroless tin plating reaction to proceed. Further promotion of the reaction was also observed upon the addition of dimethylthiourea. Our results therefore indicated that a ChCl-urea/thiourea IL-based electrolyte containing malonic acid, dimethylthiourea, sodium phosphate, and water can be employed as an environmentally friendly electrolyte for the electroless tin plating process with a rapid plating rate. Although further improvements are required to produce a reliable, high quality plating layer, our results demonstrate 
the feasibility of using ILs in rapid electroless tin plating to create thick and compact Sn layers.

\section{ACKNOWLEDGEMENT}

Part of the work was principally supported by the technology Innovation new development program (S2311612) funded by the Ministry of SMEs and Startups.

\section{REFERENCES}

1. C. H. Lee, S. C. Lee, and J. J. Kim, Electrochem. Solid-State Lett. 8, C110 (2005).

2. C. H. Lee, A. R. Kim, S. K. Kim, H. C. Koo, S. K. Cho, and J. J. Kim, Electrochem. Solid-State Lett. 11, D18 (2008).

3. F. A. Lowenheim and R. M. Macintosh, Modern Electro plating, 4th ed., M. Schlesinger and M. Paunovic, Editors, John Wiley \& Sons Inc., New York, pp. 242 (2000).

4. M. Arra, D. Shangguan, D. Xie, and J. Sundelio, J. Electron. Mater. 33, 977 (2004) .

5. H. Leidecker : Electrical Failure of an Accelerator Pedal Position Sensor Caused by a Tin Whisker and Discussion of Investigative Techniques Used for Whisker Detection, Proc. 5th International Symposium on Tin Whisker,
Maryland, University of Maryland, p. 14 (2011).

6. J. Zhao, N. Li, G. Cui, and J. Zhao, J. Electrochem. Soc. 153, C848 (2006).

7. P. Wasserscheid and W. Keim, Angew. Chem. Int. Ed. 39, 3772 (2000).

8. P. J. Dyson, Transition Met. Chem. 27, 353 (2002).

9. A. Ispas and A. Bund, Electrochem. Soc. Inter. Spring. 23, 47 (2014).

10. N. D. Khupse and A. Kumar, Indian J. Chem. 49, 635 (2010).

11. J. Sun, D. R. MacFarlane, and M. Forsyth, Electrochim. Acta. 48, 1707 (2003).

12. W. Li, Z. Zhang, B. Han, S. Hu, Y. Xie, and G. Yang, J. Phys. Chem. B. 111, 6452 (2007).

13. F. Endres, and S. Z. E. Abedin, Phys. Chem. Chem. Phys. 8 2101 (2006).

14. A. P. Abbott and K. J. McKenzie, Phys. Chem. Chem. Phys. 8, 4265 (2006).

15. C. Du, B. Zhao, X. B. Chen, N. Birbilis, and H. Yang, Sci. Rep. 6, 29225 (2016).

16. S. Saito, M. Lee, and W. Y. Wen, J. Am. Chem. Soc. 88 , 5107 (1966).

17. Y. Wang, J. He, W. Wang, M. Naotoshi, and Z. Chen, J. Electrochem. Soc. 160, D295 (2013). 